\section{Case Reports in Ophthalmology}

\title{
The First Argentinian Family with Familial Amyloidosis of the Finnish Type
}

\author{
Francisco Lucero Saáa Federico Andrés Cremona ${ }^{a}$ \\ Natalia Ximena Mínguez ${ }^{a} \quad$ María Laura Igarzabal $^{b} \quad$ Pablo Chiaradía $^{a}$ \\ ${ }^{a}$ Department of Ophthalmology, Hospital de Clínicas José de San Martin, Universidad de \\ Buenos Aires (UBA), Buenos Aires, Argentina; ${ }^{b}$ Centro de Educación Médica e \\ Investigaciones Clínicas Norberto Quirno (CEMIC), Buenos Aires, Argentina
}

\section{Keywords}

Familial amyloidosis, Finnish type · Lattice corneal dystrophy · Corneal pathology · Meretoja syndrome · Gelsolin

\begin{abstract}
Familial amyloidosis of the Finnish type or Meretoja syndrome is a rare autosomic dominant inherited systemic condition. It was first described by Meretoja in Finland in 1969. It is a disease produced by a single mutation in the gene coding for gelsolin, which generates an abnormal protein that cumulates in tissues and leads to various signs. Obtaining an early diagnosis can be challenging, as the first manifestations of the disease are ophthalmological and may only be seen with slit-lamp biomicroscopy. We present the first 3 cases diagnosed in Argentina, confirmed by genetic molecular testing.

(C) 2017 The Author(s)

Published by S. Karger AG, Basel
\end{abstract}

\section{Introduction}

Familial amyloidosis of the Finnish type (FAF) or Meretoja syndrome is a disease of autosomal dominant inheritance which is mainly characterized by ocular, neural, and cutaneous deposition of amyloid. It was first described by Meretoja in Finland (1969), where the pathology presents its highest prevalence. It has also been described in other European [1, 2] 


\section{Case Reports in Ophthalmology}

countries and the United States [3]. Until recently, it had been considered rare in other regions, yet new cases have been reported from all around the world, including Brazil [4], India [5], and Korea [6].

The disease develops by the presence of a mutation in the gene coding for gelsolin (GSN), located on chromosome 9q33-34. GSN is a protein involved in several processes associated with inflammation, cell motility, apoptosis, and carcinogenesis. There are two varieties, cytoplasmic and plasmatic, and the latter has been shown to be responsible for the development of the pathology [7]. The extracellular accumulation of mutant protein leads to the clinical findings of the disease, including secondary lattice lines in corneal stroma, progressive cranial and peripheral neuropathy, and cutis laxa. Amyloid deposition within corneal stroma is the earliest manifestation of the disease. Dry-eye symptoms, glaucoma, cataract, and retinitis pigmentosa have also been described, as well as systemic compromise, including carpal tunnel syndrome, autonomic nerve dysfunction, and cardiac and renal involvement [8].

\section{Case Reports}

\section{Case 1}

The proband was a 59-year-old female of Russian Ashkenazi Jewish ancestry, with a family history of an unknown disease associated with neurological symptoms and premature death. Her symptoms consisted in bilateral facial palsy, weakness, changes in personality, and decreased visual acuity that had been progressing slowly over the past 30 years. She had been referred to the Ophthalmology Division at Hospital de Clinicas in Buenos Aires for diagnosis and treatment. Clinical examination showed orthostatic hypotension, bilateral facial palsy, right lateral rectus palsy, and facial, corneal and limb hypoesthesia. Electrophysiological studies revealed bilateral facial nerve dysfunction. She had a visual acuity of 20/400 in her right eye and 20/200 in her left eye. On slit-lamp biomicroscopy, thin linear refractile lines were found in corneal stroma, arranged in a branching pattern spreading centripetally from the limbus. Mainly the periphery of the cornea was affected, while the center was spared. All these findings were consistent with secondary lattice lines due to amyloid deposition and led to the clinical diagnosis of FAF. Other findings included leukoma, corneal neovascularization, lagophthalmos, and ectropion; dry-eye evaluation showed diminished tear film break-up time and Schirmer test (Fig. 1, Fig. 2). Intraocular pressure was $15 \mathrm{~mm} \mathrm{Hg}$ and fundus examination was normal in both eyes.

\section{Cases 2, 3}

The proband's sister and son were also examined. She was 48 years old and had a history of dry-eye symptoms and lattice lines on slit-lamp biomicroscopy. She presented also with a mild right facial palsy. Her visual acuity was 20/30 OU. The proband's son was 21 years old, with no known history of ocular disease or complaints. He presented with a visual acuity of 20/20 OU. On slit-lamp biomicroscopy, no signs of amyloid deposition were found.

The patient, her sister, and 3 of her sons underwent molecular genetic testing that involved bidirectional Sanger sequencing using genomic DNA of all coding exons of the GSN gene plus $\sim 20 \mathrm{bp}$ of flanking noncoding DNA on each side. DNA was extracted from the patient specimen using the Chemagic MSM1 robot (Perkin Elmer) and quantified using SyBR Green, and quality was assessed using a Fragment Analyzer (Advanced Analytical Technologies). PCR was used to amplify the GSN exons plus additional flanking noncoding sequence. 


\section{Case Reports in Ophthalmology}

After cleaning of the PCR products, cycle sequencing was carried out using the ABI Big Dye Terminator v.3.0 kit. Products were resolved by electrophoresis on an ABI 3730xl capillary sequencer. In most cases, sequencing was performed in both forward and reverse directions; in some cases, sequencing was performed twice in either the forward or reverse directions. In nearly all cases, the full coding region of each exon as well as 20 bases of noncoding DNA flanking the exon were sequenced. Afterwards, the patient's GSN sequences were compared with the reference sequences. The outcome was that the patient, one of her sons, and her sister are heterozygous in the GSN gene for a sequence variant designated G640A (p.Asp214Asn) (Fig. 3). This variant, previously described as G654A (p.Asp187Asn), has been reported to be causative of FAF.

Even though prognosis is known to be poor, and because of her low visual acuity, a corneal transplant (penetrating keratoplasty) was the chosen treatment for the proband. First, she underwent a tarsal strip and tarsorrhaphy in her right eye, which improved both her symptoms and ocular surface. Three months later, a penetrating keratoplasty was performed. Histopathology revealed deposits of amyloid in corneal stroma. Postoperative complications in a follow-up period of 6 months included recurrent epithelial erosions.

\section{Discussion}

We present the first Argentinian family diagnosed with FAF, confirmed by molecular genetic testing. Two types of mutations have been shown to cause this disease. The first produces a transition of guanine to adenine at position 654 (G654A). This is the most common mutation and leads to a substitution of aspartic acid by asparagine at codon 187 [9]. The second mutation consists of a transition of guanine to thymine (G654T), which produces the replacement of aspartic acid by tyrosine and it is known as the Danish type of familial amyloidosis [10].

The proband showed phenotypic manifestations of FAF and also had a right lateral rectus palsy, a feature that, as far as we know, had not been previously reported as part of the disease.

Treatment consists mainly in severe dry-eye control and intraocular pressure monitoring. In some cases that present with extremely low visual acuity due to corneal scarring, a corneal transplant could be performed, but prognosis is very poor [11]. Even though a cure for the disease has not been discovered yet, there are animal models that could help develop a therapy in the future [12].

To our knowledge, this is the first case report of FAF in Argentina, but it is possible that the disease has been misdiagnosed. Bearing in mind that ocular symptoms are the first to arise, clinicians and ophthalmologists should be vigilant and look for secondary lattice lines due to amyloid deposition in patients that suffer from facial palsy. An early diagnosis by a trained ophthalmologist would help avoid future complications, consult other specialists if needed, and provide genetic counseling.

\section{Acknowledgements}

The authors are grateful to Prevention Genetics (Marshfield, WI, USA) for their help with the genetic testing of our patients. 
Lucero Saá et al.: The First Argentinian Family with Familial Amyloidosis of the Finnish Type

Statement of Ethics

Subjects described in this case series have given their informed written consent and agree on the publishing of the article.

\section{Disclosure Statement}

None of the authors has any conflicts of interest in any aspects of this report.

\section{References}

-1 Conceicao I, Sales-Luis ML, De Carvalho M, et al: Gelsolin-related familial amyloidosis, Finnish type, in a Portuguese family: clinical and neurophysiological studies. Muscle Nerve 2003;28:715-721.

-2 Huerva V, Velasco A, Sanchez MC, Mateo AJ, Matias-Guiu X: Lattice corneal dystrophy type II: clinical, pathologic, and molecular study in a Spanish family. Eur J Ophthalmol 2007;17:424-429.

- 3 Kiuru S: Gelsolin-related familial amyloidosis, Finnish type (FAF), and its variants found worldwide. Amyloid 1998;5:55-66.

4 Solari HP, Ventura MP, Antecka E, et al: Danish type gelsolin-related amyloidosis in a Brazilian family: case reports. Arq Bras Oftalmo 2011;74:286-288.

5 Maramattom BV, Chickabasaviah YT: A new Indian family affected by gelsolin amyloidosis. Neurol India 2013;61:673-675.

-6 Park KJ, Park JH, Park JH, Cho EB, Kim BJ, Kim JW: The first Korean family with hereditary gelsolin amyloidosis caused by p.D214Y mutation in the GSN gene. Ann Lab Med 2016;36:259-262.

7 Kangas H, Paunio T, Kalkkinen N, et al: In vitro expression analysis shows that the secretory form of gelsolin is the sole source of amyloid in gelsolin-related amyloidosis. Hum Mol Genet 1996;5:12371243.

8 Ardalan MR, Shoja MM, Kiuru-Enari S: Amyloidosis-related nephrotic syndrome due to a G654A gelsolin mutation: the first report from the Middle East. Nephrol Dial Transplant 2007;22:272-275.

-9 Levy E, Haltia M, Fernandez-Madrid I, et al: Mutation in gelsolin gene in Finnish hereditary amyloidosis. J Exp Med 1990;172:1865-1867.

10 Maury CP, Liljeström M, Boysen G, et al: Danish type gelsolin related amyloidosis: 654G-T mutation is associated with a disease pathogenetically and clinically similar to that caused by the 654G-A mutation (familial amyloidosis of the Finnish type). J Clin Pathol 2000;53:95-99.

11 Mattila JS, Krootila K, Kivelä T, et al: Penetrating keratoplasty for corneal amyloidosis in familial amyloidosis, Finnish type. Ophthalmology 2015;122:457-463.

12 Solomon JP, Page LJ, Balch WE, et al: Gelsolin amyloidosis: genetics, biochemistry, pathology and possible strategies for therapeutic intervention. Crit Rev Biochem Mol Biol 2012;47:282-296. 
Case Reports in
Ophthalmology

Case Rep Ophthalmol 2017;8:446-451

\begin{tabular}{l|l}
\hline DOI: $10.1159 / 000479729$ & C 2017 The Author(s). Published by S. Karger AG, Basel \\
\hline
\end{tabular} www.karger.com/cop

Lucero Saá et al.: The First Argentinian Family with Familial Amyloidosis of the Finnish Type

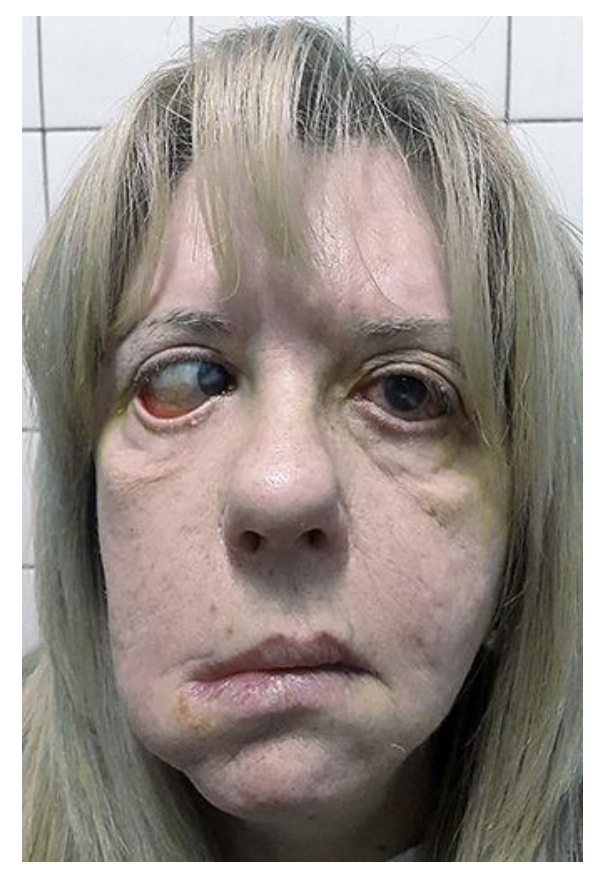

Fig. 1. Proband. Bilateral facial palsy, leukoma, lagophthalmos, ectropion, and right lateral rectus palsy.

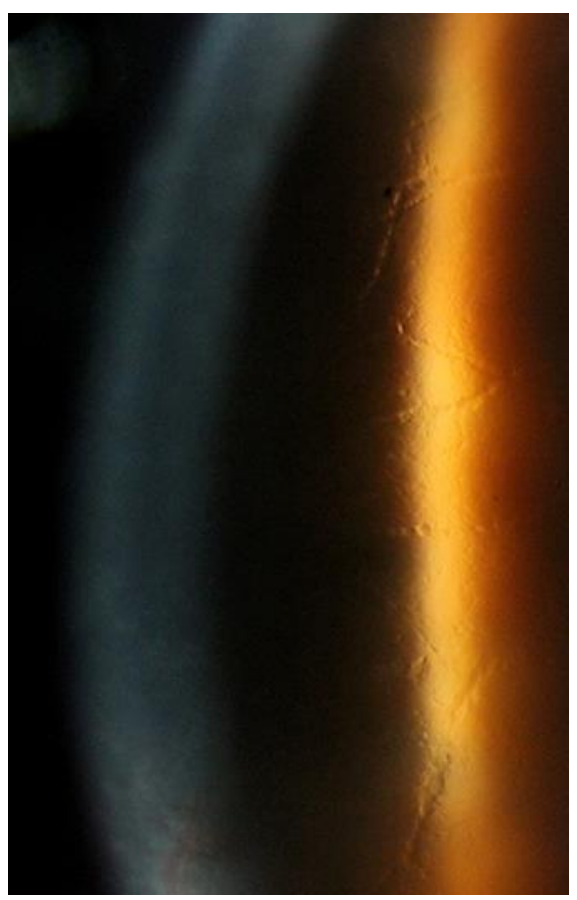

Fig. 2. Corneal lattice dystrophy, as seen in slit-lamp biomicroscopy. 


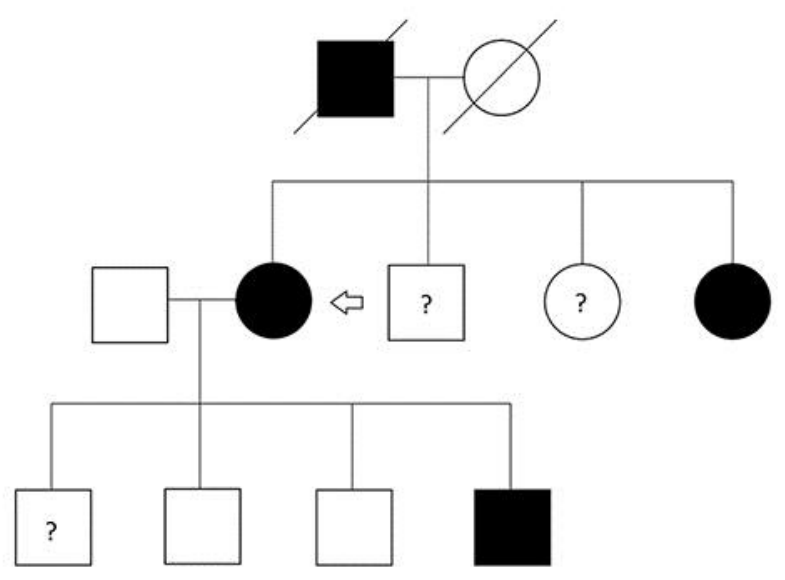

Fig. 3. Pedigree of the Argentinian family with hereditary gelsolin amyloidosis. The proband is represented with an arrow. Affected members are shown in black. The proband's first son had symptoms but could not be tested. 